\section{Statin use and tooth loss in chronic periodontitis patients}

Cunha-Cruz J, Saver B et al. J Periodonto/ 2006; 77: 1061-1066

Statin use over a $3 \mathrm{yr}$ period was not consistently associated with reduced tooth loss in the 4 th yr and subsequently.

Statins have anti-inflammatory and bone stimulating effects which may be of periodontal importance. In this retrospective study from NW USA, statin use was examined in relation to tooth loss in a group of 1,021 patients followed for a mean of 7.1 yrs. Subjects were members of a health maintenance organisation with medical, dental and pharmaceutical benefit provision.

After adjustment for baseline variation, use of antibiotics and other anti-inflammatory agents, and periodontal treatment, any use of statins over any $3 \mathrm{yr}$ period was not associated with tooth loss in the subsequent 4th year. Regular statin use was also not associated, although tooth loss was decreased by $37 \%$ in the 4 th year $(\mathrm{RR}=$ 0.63; CI: 0.32-1.25). However, any statin use during the 3 yrs after the initial periodontal exam was significantly associated with $48 \%$ decreased tooth loss in the 4 th and subsequent years $(\mathrm{RR}=0.52$; $\mathrm{CI}$ : 0.29-0.95). The authors consider further studies are indicated.

doi:10.1038/sj.bdj.4814119

\section{LOCAL ANAESTHESIA}

\section{Articaine and lidocaine mandibular buccal infiltration anesthesia: a prospective randomized double-blind cross-over study}

Kanaa MD, Whitworth JM et al. J Endod 2006; 32: 296-298

Articaine infiltration was more successful in this region.

Articaine (AC) is a recently introduced local anaesthetic agent which has similar reported effects to lidocaine (LC). Infiltration anaesthesia is not normally used for pulpal anaesthesia in lower molars.

The present study compared AC 4\% and LC 2\% (each with $1: 100,000$ epinephrine) in a double-blind cross-over trial involving 31 healthy student subjects (mean age 23 yrs; 15 male), who were randomised to $1.8 \mathrm{ml}$ of each drug on two occasions at least 1 week apart, on the same first molar. An electronic tester was used to determine pulp vitality before injection, and anaesthesia at stimulation up to a maximum $80 \mu \mathrm{A}$, at 2 min intervals afterwards up to 30 min.

After injection, 65\% of AC and 38\% of LC infiltrations led to absence of pulpal sensation $(P<0.01)$, respectively in 20 and 12 subjects $(P<0.01)$. The total numbers of negative tests was 236 for $\mathrm{AC}$ and 129 for $\mathrm{LC}(P<0.001)$. With AC, 6 subjects were negative at all tests, and with LC, 2 . All subjects reported lip numbness with both injections.

\section{Foetal alcohol syndrome: a dental and skeletal age analysis of patients and controls}

Naidoo S, Norval G et al. Eur J Orthod 2006; 28: 247-253

In a group of children diagnosed with foetal alcohol syndrome (FAS), skeletal development appeared delayed, but dental development was significantly delayed only in boys.

FAS is a condition resulting from significant maternal alcohol intake during pregnancy, and may result in facial abnormalities, CNS dysfunction, growth deficiency and other effects. In this study, 45 girls and 45 boys diagnosed with FAS were compared with controls matched for age, gender and social class.

Mean chronological ages of subjects and controls were 9.04 and 8.95 yrs, respectively. Respective mean dental ages of boy subjects and controls were 9.1 and 9.96 yrs, and of girls, 8.33 and 8.79. Respective mean skeletal ages of boy subjects and controls were 7.0 and 8.3 yrs, and of girls, 8.1 and 8.9. Differences in delay scores were statistically significant except for dental age in girls.

doi:10.1038/sj.bdj.4814120

\section{ENDODONTICS}

\section{Respective evaluation of surgical} endodontic treatment: traditional versus modern technique

J Endod 2006; 32: 412-416

Success with the modern technique was twice that with the traditional technique.

Traditional surgical endodontic technique involves apicectomy and retrograde root filling (RRF) using a bur. Recently, a modern technique has been developed, using an operating microscope, minimal apical resection and ultrasonic RRF. Respective success rates of $60 \%$ and $90 \%$ have been reported. In this study, records for 43 teeth in 36 patients who had received the traditional approach were compared retrospectively with those for 45 teeth in 35 patients who had received the modern technique.

Success after a mean 11 months' follow-up was measured as either a complete apical cavity bone fill or scar tissue healing. Treatment had a successful outcome in 41 teeth receiving the modern approach, but in only 19 with the traditional approach, an uncertain outcome respectively in 2 and 5 , and failure (unchanged or enlarged radiolucency) in 2 and $19(P<0.0001)$. The only other difference found between the groups was the age of subjects (mean 36 yrs for traditional, 44 yrs for modern; $P<0.05$ ). Tooth type was associated with failure (in mandibular posteriors) when the traditional approach was used $(P<0.05)$. 